\title{
FAT SETS AND SATURATED IDEALS
}

\author{
JOHN KRUEGER
}

\begin{abstract}
We strengthen a theorem of Gitik and Shelah [6] by showing that if $\kappa$ is either weakly inaccessible or the successor of a singular cardinal and $S$ is a stationary subset of $\kappa$ such that $N S_{\kappa} \mid S$ is saturated then $\kappa \backslash S$ is fat. Using this theorem we derive some results about the existence of fat stationary sets. We then strengthen some results due to Baumgartner and Taylor [2], showing in particular that if $I$ is a $\lambda^{+++}$-saturated normal ideal on $P_{\kappa} \lambda$ then the conditions of being $\lambda^{+}$-preserving, weakly presaturated, and presaturated are equivalent for $I$.
\end{abstract}

$\S 1$. Introduction. In this paper we strengthen several known theorems about saturated and presaturated ideals, and present some results about the existence of fat stationary sets. One of the better known results in the area of saturated ideals is the following theorem of Gitik and Shelah [6]: if $\theta<\theta^{+}<\kappa$ are regular cardinals then $N S_{\kappa} \uparrow \operatorname{cof}(\theta)$ is not saturated. This theorem solved the problem of whether $N S_{\kappa}$ can be saturated for $\kappa$ weakly inaccessible. In Section 3 we strengthen this theorem by proving that if $\kappa$ is either weakly inaccessible or the successor of a singular cardinal and $S$ is a stationary subset of $\kappa$ such that $N S_{\kappa}\lceil S$ is saturated then $S$ is co-fat. Using this theorem we prove some results about fat stationary sets. In particular we prove that if $\kappa$ is weakly inaccessible or the successor of a singular cardinal then any stationary subset of $\kappa$ contains a stationary co-fat subset.

In the fourth section we discuss presaturated ideals and strengthen some results of Baumgartner and Taylor [2]. The idea of a presaturated ideal was introduced in [2] as a generalization of saturation. They proved that if $I$ is a normal $\lambda^{++}$-saturated ideal on $P_{\kappa} \lambda$ then the conditions of being $\lambda^{+}$-preserving, weakly presaturated, and presaturated are equivalent for $I$. We strengthen this result by showing that if $I$ is $\lambda^{+++}$-saturated then the three conditions are equivalent. In particular, if $I$ is a normal ideal on a regular cardinal $\kappa$ and $2^{\kappa} \leq \kappa^{++}$, or if GCH holds and $I$ is a normal ideal on $P_{\kappa} \lambda$, then the conditions are equivalent for $I$.

This paper forms a part of my Ph.D. dissertation under the supervision of James Cummings. 
§2. Preliminaries. If $\kappa$ is regular and $\kappa \leq \lambda$, we define $P_{\kappa} \lambda=\{a \subseteq \lambda$ : $|a|<\kappa, a \cap \kappa \in \kappa\} .{ }^{1}$ Note that $P_{\kappa} \kappa=\kappa$. A subset of $P_{\kappa} \lambda$ is club if it is closed under unions of increasing sequences of length less than $\kappa$ and is cofinal; a set is stationary if it intersects every club. The ideal of non-stationary subsets of $P_{\kappa} \lambda$ is denoted by $N S_{\kappa, \lambda}$ (or $N S_{\kappa}$ if $\kappa=\lambda$ ). If $S$ is stationary, $N S_{\kappa, \lambda} \uparrow S$ is the ideal generated by $N S_{\kappa, \lambda}$ along with the complement of $S$. An ideal $I$ on $P_{\kappa} \lambda$ is fine if for each $\xi<\lambda$ the set $\left\{a \in P_{\kappa} \lambda: \xi \notin a\right\}$ is in $I$. In what follows if we say that $I$ is an ideal we mean that $I$ is a $\kappa$-complete fine ideal on some space $P_{\kappa} \lambda$.

An antichain of stationary subsets of $P_{\kappa} \lambda$ is a family of stationary sets any two of which have non-stationary intersection. If $I$ is an ideal on $P_{\kappa} \lambda, I^{+}$is the forcing poset consisting of subsets of $P_{\kappa} \lambda$ which are not in $I$, ordered by inclusion. An ideal $I$ on $P_{\kappa} \lambda$ is $\mu$-saturated if there is no antichain of $I^{+}$of size $\mu$, and $I$ is saturated if it is $\lambda^{+}$-saturated. An ideal of the form $N S_{\kappa, \lambda} \uparrow S$ is saturated if and only if there is no antichain of stationary subsets of $S$ of size $\lambda^{+}$. The saturation of an ideal $I$, denoted $\operatorname{sat}(I)$, is the least cardinal $\mu$ such that $I$ is $\mu$-saturated. We assume familiarity with generic ultrapowers in Section 4. The forcing poset $I^{+}$adds a filter $U$ which measures every subset of $P_{\kappa} \lambda$ in $V$, and induces a generic embedding $j: V \rightarrow M$ where $M$ is the ultrapower of $V$ by $U$. We say that $I$ is precipitous if $I^{+}$forces that $M$ is well-founded. If $I$ is a saturated ideal on $P_{\kappa} \lambda$ then $I$ is precipitous, $I^{+}$forces that $\lambda^{+}$is a cardinal, and $I^{+}$forces that the generic ultrapower is closed under $\lambda$-sequences.

We reserve the letters $\kappa, \lambda$, and $\mu$ for infinite cardinals, and the letter $n$ for natural numbers. The term $\operatorname{cof}(\theta)$ indicates the class of ordinals with cofinality $\theta$, and similarly for $\operatorname{cof}(\geq \theta)$ and $\operatorname{cof}(>\theta)$. We use the fact that if $\kappa$ is regular and $S$ is a stationary set of singular ordinals then there is a regular $\theta<\kappa$ such that $S \cap \operatorname{cof}(\theta)$ is stationary (just apply Fodor's Lemma). We denote the class of singular limit ordinals by Sing and the class of regular cardinals by Reg. If $\mathbb{P}$ is a forcing poset and $\lambda$ is a cardinal, terms of the form $\lambda^{+n}$ in forcing statements indicate $\lambda^{+n}$ as computed in the ground model.

§3. Fat sets and saturated ideals. A fat subset of a regular uncountable cardinal $\kappa$ is a set $A$ such that for any club $C$ in $\kappa, A \cap C$ has closed subsets of any order type less than $\kappa$. Thus fatness is intermediate between stationarity and clubness. A set $A$ is co-fat if $\kappa \backslash A$ is fat. In this section we prove that if $\kappa$ is either weakly inaccessible or the successor of a singular cardinal and $S$ is a stationary subset of $\kappa$ such that $N S_{\kappa} \uparrow S$ is saturated then $S$ is co-fat. A slightly weaker result holds for successors of regulars.

We proved this last theorem in an effort to answer the following combinatorial question about fat sets: is it provable in ZFC that there exist non-trivial fat sets in a regular uncountable cardinal? There are some standard examples of fat stationary sets. Any club set is fat. It is not difficult to see that if $\kappa$ is weakly inaccessible then the set $\kappa \cap$ Sing is fat. We show below that if $\kappa=\mu^{+}$where $\mu$ is regular and if $A$ is contained in $\operatorname{cof}(\mu)$ and $\operatorname{cof}(\mu) \backslash A$ is stationary, then the complement of $A$ is a fat set. Also, a set which almost contains any such set

\footnotetext{
${ }^{1}$ Usually $P_{\kappa} \lambda$ is used to denote the set $[\lambda]^{<\kappa}=\{a \subseteq \lambda:|a|<\kappa\}$. The set $P_{\kappa} \lambda$ as we define it is a club subset of $[\lambda]^{<\kappa}$ which is easier to work with than $[\lambda]^{<\kappa}$.
} 
modulo clubs is fat. We refer to these sets as trivially fat. We asked whether or not it is provable in ZFC that there exist non-trivial fat sets in a regular uncountable cardinal.

Our main result on fat sets is that if $\kappa$ is weakly inaccessible or the successor of a singular cardinal then any co-stationary set $B$ can be enlarged to a set $A$ which is fat but not club. In other words, any stationary set $S$ can be thinned out to a stationary co-fat set. This result solves the question about the existence of nontrivial fat sets for these two sorts of cardinals as follows: if $S$ is a stationary co-fat subset of Sing, then $S$ has a non-trivial fat complement. For the case $\kappa=\mu^{+}$ where $\mu$ is regular uncountable the question about the existence of non-trivial fat sets in $\kappa$ is independent of ZFC.

The main use of fat sets in the literature thus far has been variations of the following theorem: under $\mathrm{GCH}$, if $\kappa$ is regular and $A$ is stationary in $\kappa$ then $A$ is fat if and only if there is a $\kappa$-distributive forcing poset $\mathbb{P}$ such that $\mathbb{P}$ forces that $A$ contains a club (see [1]). In the present paper we are interested in fat sets in their own right rather than in applications of this theorem.

The following lemma explains what fat subsets of successor cardinals are like. The first statement of the lemma appears in [1].

LEMMA 1. Let $\kappa=\mu^{+}$where $\mu$ is a cardinal.

1. Suppose that $\mu$ is regular. A set $A$ is fat in $\kappa$ if for any club $C$, the set $A \cap C$ contains a closed subset of order type $\mu+1$.

2. Suppose that $\mu$ is singular. A set $A$ is fat in $\kappa$ if for any club $C$, the set $A \cap C$ contains closed subsets of any order type less than $\mu$.

Proof. We prove 1 and 2 simultaneously. We show by induction that for all $\xi<\kappa$ and for any club $C$, the set $A \cap C$ contains a closed subset of order type $\xi+1$. We can assume $\xi \geq \mu$. If $\xi$ is a successor ordinal then the statement follows from induction and the fact that $A$ is stationary. Suppose that $\xi$ is a limit ordinal and the claim holds below $\xi$. Let $C$ be club. Define $E$ as the club of $\alpha$ less than $\kappa$ such that for all $\beta<\alpha$ and for all $\zeta<\xi$, the set $A \cap C \cap(\beta, \alpha)$ contains a closed subset with order type $\zeta+1$. Let $\theta=\operatorname{cf}(\xi)$. Then either $\theta<\mu$, or if $\mu$ is regular possibly $\theta=\mu$. In either case there is a closed subset of $A \cap C \cap E$ of order type $\theta+1$, which we enumerate as $\left\langle\alpha_{i}: i \leq \theta\right\rangle$. Let $\left\langle\xi_{i}: i<\theta\right\rangle$ be an increasing sequence unbounded in $\xi$. For each $i<\theta$ fix a closed set $c_{i}$ with order type $\xi_{i}+1$ contained in $A \cap C \cap\left(\alpha_{i}, \alpha_{i+1}\right)$. Let $d$ be the union of all the $c_{i}$ 's along with all the $\alpha_{i}$ 's. Then $d$ is a closed subset of $A \cap C$ with order type at least $\xi+1$.

A consequence of this lemma is that every stationary subset of $\omega_{1}$ is fat. For let $S$ be stationary in $\omega_{1}$ and suppose that $C$ is club. Then $S$ intersects $\lim (S \cap C)$ at some point $\alpha$. The set $S \cap C$ is unbounded in $\alpha$ and $\alpha$ is in $S \cap C$, so there is a closed subset of $S \cap C$ with order type $\omega+1$.

The following is a useful characterization of co-fatness in terms of stationary reflection. The proof is straightforward.

Lemma 2. Suppose that $\kappa$ is weakly inaccessible or $\kappa=\mu^{+}$where $\mu$ is singular. Let $A$ be a subset of $\kappa$ and let $T$ be the set of limit ordinals $\alpha$ less than $\kappa$ with uncountable cofinality such that $A \cap \alpha$ is non-stationary in $\alpha$. The following are 
equivalent.

1. A is co-fat.

2. For all regular uncountable cardinals $\theta<\kappa$, the set $T \cap \operatorname{cof}(\theta)$ is stationary.

3. There are unboundedly many regular cardinals $\theta<\kappa$ such that $T \cap \operatorname{cof}(\theta)$ is stationary.

The following proposition is our main tool for relating fatness to saturation.

Proposition 1. Let $\kappa$ be regular and let $A$ be a fat subset of $\kappa$. Suppose that $\mathcal{A}$ is an antichain of stationary sets $B \subseteq A$ such that $A \backslash B$ is not fat. If $\kappa$ is weakly inaccessible then $|\mathcal{A}| \leq \kappa$. If $\kappa=\mu^{+}$and $\mu$ is a singular cardinal then $|\mathcal{A}| \leq \mu$.

Proof. Let $\delta$ be equal to $\kappa$ or $\mu$ depending on whether $\kappa$ is inaccessible or the successor of a singular cardinal $\mu$. Suppose for a contradiction that there is an antichain $\mathcal{A}$ as above with size $\delta^{+}$. Enumerate $\mathcal{A}$ as $\left\langle A_{i}: i<\delta^{+}\right\rangle$. Since $A \backslash A_{i}$ is not fat, its complement $A_{i} \cup(\kappa \backslash A)$ is not co-fat. For each $i$ let $T_{i}$ be the set of $\alpha \in \kappa \cap \operatorname{cof}(>\omega)$ such that $\left(A_{i} \cup(\kappa \backslash A)\right) \cap \alpha$ is non-stationary in $\alpha$. By the previous lemma, for each $i$ there is a regular uncountable cardinal $\theta_{i}<\kappa$ such that $T_{i} \cap \operatorname{cof}\left(\theta_{i}\right)$ is non-stationary. It is easy to see by cases that there is a set $Z \subseteq \delta^{+}$with size $\delta^{+}$such that $\theta_{i}=\theta$ for all $i \in Z$. Choose a set $Y$ contained in $Z$ with order type $\theta^{+}$. Note that $\theta^{+}<\kappa$. Now for each $i<j$ in $Y$ choose a club $C(i, j)$ such that $A_{i} \cap A_{j} \cap C(i, j)$ is empty. For each $i$ in $Y$ choose a club $D_{i}$ such that $T_{i} \cap \operatorname{cof}(\theta) \cap D_{i}$ is empty. Now let $C=\bigcap\{C(i, j): i<j$ in $Y\} \cap \bigcap\left\{D_{i}: i \in Y\right\}$. Since $|Y|=\theta^{+}<\kappa, C$ is club.

Applying the fatness of $A$ choose a closed set $X$ contained in $A \cap C$ with order type $\theta+1$, and let $\alpha=\sup (X)$. Note that $\alpha$ is in $C \cap \operatorname{cof}(\theta)$. For each $i$ in $Y$ the ordinal $\alpha$ is in $D_{i} \cap \operatorname{cof}(\theta)$, and therefore is not in $T_{i}$. So $\left(A_{i} \cup(\kappa \backslash A)\right) \cap \alpha$ is stationary in $\alpha$. Since $X$ is contained in $A$, it is easy to see that $A_{i} \cap X$ is stationary in $\alpha$. Moreover, if $i<j$ in $Y$ then $A_{i} \cap A_{j} \cap X \subseteq A_{i} \cap A_{j} \cap C(i, j)=\emptyset$. Thefore $\left\{A_{i} \cap X: i \in Y\right\}$ is a pairwise disjoint collection of $\theta^{+}$many non-empty subsets of $X$. This is a contradiction since $X$ has size $\theta$.

COROLlary 1. Suppose that $\kappa=\mu^{+}$where $\mu$ is a singular cardinal and $A$ is a fat subset of $\kappa$. Then any stationary set $B \subseteq A$ contains a stationary set $B_{0}$ such that $A \backslash B_{0}$ is a fat set. In particular, any stationary subset of $\kappa$ contains a stationary co-fat subset.

Proof. Let $A$ be fat and $B \subseteq A$ stationary. Fix a sequence $\left\langle B_{i}: i<\kappa\right\rangle$ which partitions $B$ into pairwise disjoint stationary sets. Since the partition has size greater than $\mu$, by the last proposition there is $i$ such that $A \backslash B_{i}$ is fat. Setting $A$ to be $\kappa$, this gives that every stationary subset of $\kappa$ contains a co-fat stationary subset.

The following has a similar proof.

Corollary 2. Suppose that $\kappa$ is weakly inaccessible and $A$ is a fat subset of $\kappa$. For any stationary set $B \subseteq A$ such that $N S_{\kappa} \uparrow B$ is not saturated, there is a stationary set $B_{0} \subseteq B$ such that $A \backslash B_{0}$ is fat. In particular any stationary subset of $\kappa$ on which the non-stationary ideal is not saturated contains a stationary cofat subset. 
In order to prove that every stationary subset of a weakly inaccessible cardinal contains a stationary co-fat subset, we strengthen the main theorem of Gitik and Shelah [6] on the non-saturation of the ideal $N S_{\kappa} \uparrow \operatorname{cof}(\theta)$ for regular $\theta<\kappa$. The following lemma describes a club guessing property which is slightly more general than a similar property which was proven in [6]. The proof is the same as the proof which appears in [6] so we omit it.

Lemma 3. Suppose that $\theta_{0} \leq \theta<\theta^{+}<\kappa$ are regular cardinals. Let $C$ be the club of limit points of the set $\kappa \cap \operatorname{cof}(>\theta)$. Suppose that $S$ is a stationary subset of $\kappa \cap \operatorname{cof}\left(\theta_{0}\right)$. If $N S_{\kappa}\left\lceil S\right.$ is saturated then there exists a sequence $\left\langle s_{\alpha}: \alpha \in S \cap C\right\rangle$ such that:

1. $s_{\alpha} \subseteq \alpha \cap \operatorname{cof}(>\theta)$,

2. $s_{\alpha}$ is unbounded in $\alpha$,

3. for any club $D$ there is a club $E$ such that for all $\alpha$ in $S \cap C \cap E$ there is $\beta<\alpha$ such that $s_{\alpha} \backslash \beta \subseteq D$.

THEOREM 1. Assume that $\kappa$ is either weakly inaccessible or $\kappa=\mu^{+}$where $\mu$ is a singular cardinal. Suppose that $S$ is a stationary subset of $\kappa$ such that $N S_{\kappa} \uparrow S$ is saturated. Then $S$ is co-fat.

Proof. The proof is similar to the proof from [6]. Suppose for a contradiction that $S$ is not co-fat. Then $S$ is not almost contained in the co-fat set Reg, so $S \cap$ Sing is stationary. Fix a regular cardinal $\theta$ less than $\kappa$ and a club $D$ such that $D \backslash S$ contains no closed subsets of order type $\theta$, and $\theta$ is large enough so that $S \cap \operatorname{cof}(<\theta)$ is stationary. Note that $\theta^{+}<\kappa$. Fix a sequence $\left\langle\theta_{i}: i<\xi\right\rangle$ which enumerates every regular cardinal $\zeta$ below $\theta$ such that $S \cap \operatorname{cof}(\zeta)$ is stationary. Let $F$ be a club such that for all $\alpha$ in $S \cap \operatorname{cof}(<\theta) \cap F$ there is $i<\xi$ such that $\operatorname{cf}(\alpha)=\theta_{i}$. For each $i<\xi$ the set $T_{i}=S \cap \operatorname{cof}\left(\theta_{i}\right)$ is stationary and $N S_{\kappa} \uparrow T_{i}$ is saturated. So fix a club guessing sequence $\left\langle s_{\alpha}^{i}: \alpha \in T_{i} \cap C\right\rangle$ as in the preceding lemma, where $C$ is the club of limit points of the set $\kappa \cap \operatorname{cof}(>\theta)$.

Define a sequence of clubs $\left\langle E_{i}: i \leq \theta\right\rangle$ as follows. Let $E_{0}=C \cap D \cap F$. Take intersections at limits. Suppose that $E_{j}$ is given. For each $i<\xi$ there is a club $F_{j}^{i}$ such that for every $\alpha$ in $T_{i} \cap C \cap F_{j}^{i}$ there is $\beta<\alpha$ such that $s_{\alpha}^{i} \backslash \beta \subseteq E_{j}$. Let $E_{j+1}=\lim \left(E_{j}\right) \cap \bigcap\left\{F_{j}^{i}: i<\xi\right\}$.

Let $\delta$ be the least member of the set $S \cap E_{\theta} \cap \operatorname{cof}(<\theta)$. Fix $i<\xi$ such that $\operatorname{cf}(\delta)=\theta_{i}$. We claim that $s_{\delta}^{i} \cap E_{\theta}$ is non-empty. Let $\left\langle\alpha_{j}: j<\theta_{i}\right\rangle$ be increasing and unbounded in $\delta$. For each $l<\theta, \delta \in F_{l}^{i} \cap T_{i} \cap E_{0}$, and therefore there is $k_{l}<\theta_{i}$ such that $s_{\delta}^{i} \backslash \alpha_{k_{l}} \subseteq E_{l}$. Let $Y$ be a set of size $\theta$ and $k$ fixed such that for all $l$ in $Y, k_{l}=k$. Then $s_{\delta}^{i} \backslash \alpha_{k} \subseteq \bigcap\left\{E_{l}: l \in Y\right\}=E_{\theta}$. So $s_{\delta}^{i} \cap E_{\theta}$ is non-empty. Choose some $\gamma$ in $s_{\delta}^{i} \cap E_{\theta}$. Then $\operatorname{cof}(\gamma)>\theta$, and $\gamma \in \lim \left(E_{l}\right)$ for all $l<\theta$, therefore $E_{\theta} \cap \gamma$ is club in $\gamma$. Let $X$ be a closed subset of $E_{\theta} \cap \gamma$ of order type $\theta$ consisting of ordinals of cofinality less than $\theta$. Since $D \backslash S$ does not contain any closed subsets of order type $\theta$, it does not contain $X$. So $X \cap S$ is non-empty. But if $\xi \in X \cap S$ then $\xi \in S \cap E_{\theta} \cap \operatorname{cof}(<\theta)$ and $\xi<\delta$, which contradicts the minimality of $\delta$.

This theorem generalizes the result of Gitik and Shelah [6] since the set $\kappa \cap$ $\operatorname{cof}(\theta)$ is not co-fat. Combining the last theorem with Lemma 2 we get the following. 
COROllary 3. Suppose that $\kappa$ is weakly inaccessible or the successor of a singular cardinal and $S$ is a stationary subset of $\kappa$ such that $N S_{\kappa} \uparrow S$ is saturated. Then for every regular uncountable cardinal $\theta<\kappa$ there is a stationary set of $\alpha$ in $\kappa \cap \operatorname{cof}(\theta)$ such that $S \cap \alpha$ is non-stationary in $\alpha$.

Corollary 4. Suppose that $\kappa$ is weakly inaccessible. Then every stationary subset of $\kappa$ contains a stationary co-fat subset.

Proof. Let $S$ be stationary. If $N S_{\kappa} \uparrow S$ is saturated then $S$ is cofat. Otherwise $S$ can be broken up into an antichain of $\kappa^{+}$many stationary subsets. By Proposition 1 one of those subsets is co-fat.

COROLlary 5. Suppose that $\kappa$ is either weakly inaccessible or the successor of a singular cardinal. Then there exist non-trivial fat subsets of $\kappa$.

Proof. Let $S$ be a stationary co-fat subset of Sing. Then the complement of $S$ is a non-trivial fat set, since it is disjoint from a stationary set of singular ordinals.

We now consider the case where $\kappa=\mu^{+}$and $\mu$ is a regular uncountable cardinal. Suppose that $A$ is a stationary subset of $\kappa$ such that $A \cap \operatorname{cof}(\mu)$ is stationary and $\operatorname{cof}(<\mu) \backslash A$ is non-stationary. Then $A$ is fat. For let $C$ be a club. Fix a club $D$ such that $D \cap \operatorname{cof}(<\mu) \subseteq A$. Choose an ordinal $\alpha$ in the set $\lim (C \cap D) \cap \operatorname{cof}(\mu) \cap A$. Then $C \cap D \cap \alpha$ is club in $\alpha$, so choose a club subset $X$ of $C \cap D \cap \alpha$ with order type $\mu$. Then $\lim (X) \cup\{\alpha\}$ is a closed subset of $A \cap C$ of order type $\mu+1$. We refer to sets such as $A$ as trivially fat.

We now prove that it is independent of ZFC whether there exist non-trivial fat subsets of the successor of a regular uncountable cardinal. In particular we prove that if $V=L$ then any stationary subset of the successor of a regular cardinal contains a stationary co-fat subset, which yields non-trivial fat sets. On the other hand assuming large cardinals we can force a model in which there is a successor of a regular cardinal which has no non-trivial fat subsets.

Proposition 2. Let $\kappa=\mu^{+}$where $\mu$ is a regular uncountable cardinal. Suppose that $\square_{\mu}$ holds. Then every stationary subset of $\kappa$ contains a stationary co-fat subset, and therefore there exist non-trivial fat subsets of $\kappa$.

Proof. Let $A$ be a stationary subset of $\kappa$. Let $B$ be a stationary subset of $A$ such that $\operatorname{cof}(\mu) \backslash B$ is stationary. It follows from $\square_{\mu}$ that $B$ contains a nonreflecting stationary subset $B_{0}$ (see [7]). We claim that $B_{0}$ is co-fat. Let $C$ be club. Fix an ordinal $\alpha$ in $\lim (C) \cap\left(\operatorname{cof}(\mu) \backslash B_{0}\right)$. Since $B_{0}$ is non-reflecting, the set $B_{0} \cap \alpha$ is non-stationary in $\alpha$. So choose a club $D$ in $\alpha$ with order type $\mu$ which is disjoint from $B_{0}$. Then $(C \cap D) \cup\{\alpha\}$ is a closed set with order type $\mu+1$ contained in $C \backslash B_{0}$. So $B_{0}$ is co-fat. Now let $A$ be any stationary co-fat subset of $\operatorname{cof}(<\mu)$. Then the complement of $A$ is non-trivially fat, since it is disjoint from a stationary subset of $\operatorname{cof}(<\mu)$.

On the other hand it is consistent relative to large cardinals that every fat subset of the successor of a regular cardinal is trivially fat. We use the following observation.

Proposition 3. Let $\kappa=\mu^{+}$where $\mu$ is a regular uncountable cardinal. The following condition is equivalent to the assertion that every fat subset of $\kappa$ is 
trivially fat: for every stationary set $S$ contained in $\kappa \cap \operatorname{cof}(<\mu)$, there is a club $C$ such that for all $\alpha$ in $C \cap \operatorname{cof}(\mu)$ the set $S \cap \alpha$ is stationary in $\alpha$.

Proof. Suppose that $\kappa$ has a non-trivial fat subset $A$. Then $B=\operatorname{cof}(<\mu) \backslash A$ is stationary. Let $C$ be club. Since $A$ is fat, choose a closed set $X$ contained in $A \cap C$ with order type $\mu$ consisting of ordinals with cofinality less than $\mu$. Let $\alpha=\sup X$. Then $\alpha$ is in $C \cap \operatorname{cof}(\mu)$, but $B \cap \alpha$ is non-stationary in $\alpha$ since it is disjoint from the club $X$. Now suppose that $\kappa$ has no non-trivial fat subsets. Let $S$ be a stationary subset of $\operatorname{cof}(<\mu)$. Then $A=\kappa \backslash S$ is not fat. Let $D$ be a club such that $A \cap D$ contains no closed subsets of order type $\mu+1$. Fix an ordinal $\alpha$ in $\lim (D) \cap \operatorname{cof}(\mu)$. Note that $\alpha$ is in $A \cap D$. If $S \cap \alpha$ is non-stationary then there is a set $X$ club in $\alpha$ with order type $\mu$ which is disjoint from $S$ and therefore contained in $A$. But then $(X \cap D) \cup\{\alpha\}$ is a closed subset of $A \cap D$ with order type $\mu+1$, which is impossible. So $S \cap \alpha$ is stationary in $\alpha$.

Magidor [8] constructed a model where every stationary subset of $\omega_{2}$ consisting of ordinals with cofinality $\omega$ reflects at almost every ordinal of cofinality $\omega_{1}$. His method easily generalizes to higher cardinals to construct models satisfying the stationary reflection property of the previous proposition. Hence it is consistent that $\kappa$ is the successor of a regular uncountable cardinal and every fat subset of $\kappa$ is trivially fat.

In spite of this independence, a weak form of the theorem about the existence of non-trivial fat subsets of successor of singular cardinals can be proven for successor of regulars. Let $\theta<\kappa$ be cardinals. A set $A$ is $<\theta$-fat in $\kappa$ if for any club $C$, the set $A \cap C$ contains closed subsets of any order type less than $\theta$. The following results are proven in the same manner as their analogues above.

Proposition 4. Let $\kappa=\mu^{+}$where $\mu$ is a regular uncountable cardinal and let $A$ be $a<\mu$-fat set. Suppose that $\mathcal{A}$ is an antichain of stationary sets $B \subseteq A$ such that $A \backslash B$ is not $<\mu$-fat. Then $|\mathcal{A}| \leq \mu$.

TheOREM 2. Let $\kappa=\mu^{+}$where $\mu$ is a regular uncountable cardinal. Suppose that $A$ is a $a$-fat subset of $\kappa$. Then any stationary set $B \subseteq A$ contains $a$ stationary set $B_{0}$ such that $A \backslash B_{0}$ is $<\mu$-fat. In particular any stationary subset of $\kappa$ contains a stationary $<\mu$-co-fat subset.

§4. Presaturated ideals. Baumgartner and Taylor [2] introduced presaturation as a weakening of saturation which enjoys some of its strong consequences. Let $\kappa \leq \lambda$ be cardinals with $\kappa$ regular and let $I$ be an ideal on $P_{\kappa} \lambda$. Then $I$ is $\lambda^{+}$-preserving if $I^{+}$forces that $\lambda^{+}$is a cardinal. The ideal $I$ is weakly presaturated if $I$ is $\lambda^{+}$-preserving and precipitous. Finally, we say that $I$ is presaturated if it satisfies the following combinatorial property: for any sequence $\left\langle\mathcal{A}_{\alpha}: \alpha<\lambda\right\rangle$ of antichains of $I^{+}$and for any $A \in I^{+}$there is $B \subseteq A$ in $I^{+}$such that for all $\alpha<\lambda,\left|\left\{S \in \mathcal{A}_{\alpha}: B \| S\right\}\right| \leq \lambda .^{2}$

\footnotetext{
${ }^{2}$ The definition of presaturation given in [2] is the same as what we have called weakly presaturated (following [3]). Baumgartner and Taylor defined this notion and proved their results only for ideals on $\omega_{1}$, but their results extend without difficulty to the general case given here.
} 
Clearly every weakly presaturated ideal is $\lambda^{+}$-preserving, and every presaturated ideal is weakly presaturated (see below). It is unknown whether $\lambda^{+}$preserving implies precipitousness, or even whether $\lambda^{+}$-preserving is equivalent to presaturation. As a partial solution to this problem, Baumgartner and Taylor proved the following two theorems: (1) if $I$ is a $\lambda^{++}$-saturated ideal on $P_{\kappa} \lambda$ then $\lambda^{+}$-preserving, weakly presaturated, and presaturated are equivalent for $I$; (2) if $I$ is $\lambda^{+\omega}$-saturated and $I^{+}$forces that $\operatorname{cf}\left(\lambda^{+n}\right)>\lambda$ for all $n>0$ then $I$ is weakly presaturated. We strengthen (1) by reducing the assumption to $I$ being $\lambda^{+++}$-saturated, and we strengthen the conclusion of (2) to get that $I$ is presaturated.

The proof of the following proposition appears in [5].

Proposition 5. Suppose that $I$ is a presaturated ideal on $P_{\kappa} \lambda$. Then $I$ is weakly presaturated, and $I^{+}$forces that if $j: V \rightarrow M$ is the well-founded generic embedding then ${ }^{\lambda} M \subseteq M$.

We will use the following theorem of Shelah [9]. ${ }^{3}$ We include a proof for the benefit of the reader.

Theorem 3 (Shelah). Suppose that $V \subseteq W$ are models of $Z F C, \kappa$ is regular in $V$, and $\kappa^{+V}=\kappa^{+W}$. Then $W$ models that $\operatorname{cf}(\kappa)=\operatorname{cf}(|\kappa|)$.

Proof. Since $\kappa$ is regular in $V$ there is a sequence $\left\langle A_{i}: i<\kappa^{+}\right\rangle$of unbounded subsets of $\kappa$ and a sequence $\left\langle g_{i}: i<\kappa^{+}\right\rangle$of functions from $\kappa$ to $\kappa$ such that for all $\alpha<\kappa^{+},\left\langle A_{i} \backslash g_{\alpha}(i): i<\alpha\right\rangle$ is a disjoint sequence of sets. Now work in $W$. Let $\mu=|\kappa|, \delta_{0}=\operatorname{cf}(\mu)$, and $\delta_{1}=\operatorname{cf}(\kappa)$. Fix an increasing sequence $\left\langle X_{i}: i<\delta_{0}\right\rangle$ of sets such that $\kappa=\bigcup X_{i}$ and each $\left|X_{i}\right|<\mu$. Suppose for a contradiction that $\delta_{0} \neq \delta_{1}$. Then it is easy to see that for all $i<\kappa^{+}$there is $\beta_{i}<\delta_{0}$ such that $A_{i} \cap X_{\beta_{i}}$ is unbounded in $\kappa$. Since $\kappa^{+}$is regular there is a set $Y \subseteq \kappa^{+}$of order type $\mu$ and $\beta<\delta_{0}$ such that $\beta_{i}=\beta$ for all $i \in Y$. Let $\alpha=\sup Y$. Then $\left\langle\left(A_{i} \cap X_{\beta}\right) \backslash g_{\alpha}(i): i \in Y\right\rangle$ is a disjoint sequence of non-empty subsets of $X_{\beta}$, contradicting that $X_{\beta}$ has size less than $\mu$.

Lemma 4. Let $\mathbb{P}$ be a forcing poset and let $\lambda<\lambda_{1}$ be cardinals. Assume that $\left\langle\mathcal{A}_{\alpha}: \alpha<\lambda\right\rangle$ is a sequence of antichains of $\mathbb{P}$ with each $\left|\mathcal{A}_{\alpha}\right| \leq \lambda_{1}$. Suppose that $p$ is in $\mathbb{P}$ and for all $q \leq p$ there is $\alpha<\lambda$ such that $\left|\left\{s \in \mathcal{A}_{\alpha}: q \| s\right\}\right|=\lambda_{1}$. Then $p \Vdash \operatorname{cf}\left(\lambda_{1}\right) \leq \lambda$.

Proof. Enumerate each $\mathcal{A}_{\alpha}$ as $\left\langle s_{i}^{\alpha}: i<\delta_{\alpha}\right\rangle$ where $\delta_{\alpha} \leq \lambda_{1}$. Define a name $\dot{f}$ so that for all $\alpha<\lambda, p \Vdash \dot{f}(\alpha)$ is the unique $\beta<\delta_{\alpha}$ such that $s_{\beta}^{\alpha} \in \dot{G}$ (if there is such $\beta$, otherwise 0$)$. I claim that $p \Vdash \operatorname{ran}(\dot{f})$ is unbounded in $\lambda_{1}$, and hence $\operatorname{cf}\left(\lambda_{1}\right) \leq \lambda$. Otherwise there is $q \leq p$ and $\delta<\lambda_{1}$ such that $q \Vdash \operatorname{ran}(\dot{f}) \subseteq \delta$. Fix $\alpha<\lambda$ such that $\left|\left\{s \in \mathcal{A}_{\alpha}: q \| s\right\}\right|=\lambda_{1}$. Then clearly $\delta_{\alpha}=\lambda_{1}$. Fix $\xi>\delta$ such that $q \| s_{\xi}^{\alpha}$. Let $t \leq q, s_{\xi}^{\alpha}$. Then $t \Vdash \dot{f}(\alpha)=\xi>\delta$, and also $t \Vdash \operatorname{ran}(\dot{f}) \subseteq \delta$, which is a contradiction.

THEOREM 4. Suppose that I is a normal ideal on $P_{\kappa} \lambda$ which is $\lambda^{+++}$-saturated. Then the properties of $\lambda^{+}$-preserving, weakly presaturated, and presaturated are equivalent for $I$.

${ }^{3}$ See [4] for a generalization of Shelah's theorem to singular cardinals. 
ProOF. We know that presaturated $\rightarrow$ weakly presaturated $\rightarrow \lambda^{+}$-preserving. So it suffices to show that if $I$ is $\lambda^{+}$-preserving then $I$ is presaturated. Suppose that $I$ is a $\lambda^{+++}$-saturated normal ideal on $P_{\kappa} \lambda$ which is $\lambda^{+}$-preserving. Then $I^{+}$is $\lambda^{+++}$-c.c., so it preserves $\lambda^{+++}$. By the theorem of Shelah above and the assumption that $I^{+}$preserves $\lambda^{+}$, it follows that $I^{+} \Vdash \operatorname{cf}\left(\lambda^{++}\right)=\operatorname{cf}\left(\left|\lambda^{++}\right|\right) \geq$ $\lambda^{+}>\lambda$

To show that $I$ is presaturated suppose that $\left\langle\mathcal{A}_{\alpha}: \alpha<\lambda\right\rangle$ is a sequence of antichains of $I^{+}$and $A \in I^{+}$. We wish to find $B \subseteq A$ in $I^{+}$such that for all $\alpha<\lambda,\left|\left\{X \in \mathcal{A}_{\alpha}: B \| X\right\}\right| \leq \lambda$. Since $I$ is $\lambda^{+++}$-saturated we can enumerate each $\mathcal{A}_{\alpha}$ as $\left\langle A_{i}^{\alpha}: i<\delta_{\alpha}\right\rangle$ for some $\delta_{\alpha} \leq \lambda^{++}$. Since $I^{+}$forces that $\operatorname{cf}\left(\lambda^{++}\right)>\lambda$, by the preceding lemma there is $B_{0} \subseteq A$ such that for all $\alpha<\lambda$ the set $\mathcal{B}_{\alpha}=\left\{X \in \mathcal{A}_{\alpha}: B_{0} \| X\right\}$ has size no more than $\lambda^{+}$. Since $I^{+} \Vdash \operatorname{cf}\left(\lambda^{+}\right)>\lambda$ again by the preceding lemma there is $B \subseteq B_{0}$ in $I^{+}$such that for all $\alpha<\lambda$, the set $\left\{X \in \mathcal{B}_{\alpha}: B \| X\right\}=\left\{X \in \mathcal{A}_{\alpha}: B \| X\right\}$ has size no more than $\lambda$.

We state consequences of this theorem in the more commonly considered cases.

Corollary 6. Suppose that $\kappa$ is regular and $2^{\kappa} \leq \kappa^{++}$. Then the properties of $\kappa^{+}$-preserving, weakly presaturated, and presaturated are equivalent for normal ideals on $\kappa$.

Corollary 7. Suppose that $\kappa$ is regular and $\kappa \leq \lambda$. Let I be a normal ideal on $P_{\kappa} \lambda$ such that $\left|I^{+}\right| \leq \lambda^{++}$. Then the properties of $\lambda^{+}$-preserving, weakly presaturated, and presaturated are equivalent for I. In particular under GCH the properties are equivalent.

We now strengthen the second theorem of [2] mentioned above: if $I$ is an ideal on $P_{\kappa} \lambda$ which is $\lambda^{+\omega}$-saturated and $I^{+} \Vdash \operatorname{cf}\left(\lambda^{+n}\right)>\lambda$ for all $n>0$, then $I$ is weakly presaturated. We strengthen this to show that under even weaker assumptions $I$ is presaturated. Note that since sat $(I)$ is regular, the assumption that $I$ is $\lambda^{+\omega}$-saturated means that there is $n$ such that $I$ is $\lambda^{+n}$-saturated.

THEOREM 5. Let I be an ideal on $P_{\kappa} \lambda$ which is $\lambda^{+n}$-saturated for some $n>1$. Assume that for all $0<m<n-1, I^{+} \Vdash \operatorname{cf}\left(\lambda^{+m}\right)>\lambda$. Then $I$ is presaturated.

Proof. Suppose that $I$ and $n$ are as in the statement of the theorem. Since $I^{+} \Vdash \operatorname{cf}\left(\lambda^{+}\right)>\lambda, I$ is $\lambda^{+}$-preserving. By Shelah's theorem, $I^{+} \Vdash \operatorname{cf}\left(\lambda^{+n-1}\right)=$ $\operatorname{cf}\left(\left|\lambda^{+n-1}\right|\right) \geq \lambda^{+}>\lambda$. To show that $I$ is presaturated let $\left\langle\mathcal{A}_{\alpha}: \alpha<\lambda\right\rangle$ be a sequence of antichains of $I^{+}$. Fix $A$ in $I^{+}$. Let $m$ be the least integer such that there is some $B_{0} \subseteq A$ in $I^{+}$such that for all $\alpha<\lambda,\left|\left\{X \in \mathcal{A}_{\alpha}: B_{0} \| X\right\}\right| \leq \lambda^{+m}$. Note that by the saturation assumption, $m \leq n-1$. Fix $B_{0} \subseteq A$ in $I^{+}$such that for all $\alpha<\lambda, \mathcal{B}_{\alpha}=\left\{X \in \mathcal{A}_{\alpha}: B_{0} \| X\right\}$ has size less than or equal to $\lambda^{+m}$. Suppose for a contradiction that $m>0$. By the minimality of $m$, for all $B \subseteq B_{0}$ in $I^{+}$, there is $\alpha<\lambda$ such that $\left|\left\{X \in \mathcal{A}_{\alpha}: B \| X\right\}\right| \geq \lambda^{+m}$; but $\left\{X \in \mathcal{B}_{\alpha}: B \| X\right\}=\left\{X \in \mathcal{A}_{\alpha}: B \| X\right\}$, and so this set has size $\lambda^{+m}$. By a previous lemma it follows that $B_{0} \Vdash \operatorname{cf}\left(\lambda^{+m}\right) \leq \lambda$, which is a contradiction.

\section{REFERENCES}

[1] U. Abraham and S. Shelah, Forcing closed unbounded sets, J. Symbolic Logic, vol. 48 (1983), no. 3, pp. 643-657. 
[2] J. Baumgartner and A. TAYlor, Saturation properties of ideals in generic extensions II, Trans. Amer. Math. Soc., vol. 271 (1982), no. 2, pp. 587-609.

[3] D. Burke and Y. Matsubara, The extent of strength in the club filters, Israel J. Math, vol. 114 (1999), pp. 253-263.

[4] J. Cummings, Collapsing successors of singulars, Proc. Amer. Math. Soc., vol. 125 (1997), no. 9, pp. 2703-2709.

[5] M. Foreman, M. Magidor, and S. Shelah, Martin's maximum, saturated ideals, and non-regular ultrafilters. part I, Annals of Mathematics, vol. 127 (1988), no. 3, pp. 1-47.

[6] M. Gitik and S. Shelah, Less saturated ideals, Proc. Amer. Math. Soc., vol. 125 (1997), no. 5, pp. 1523-1530.

[7] T. JeCh, Set theory, Springer, 1997, Second edition.

[8] M. Magidor, Reflecting stationary sets, J. Symbolic Logic, vol. 47 (1982), no. 4, pp. 755-771.

[9] S. Shelah, Cardinal arithmetic, Oxford University Press, 1994.

\section{DEPARTMENT OF MATHEMATICAL SCIENCES}

CARNEGIE MELLON UNIVERSITY

5000 FORBES AVE.

PITTSBURGH, PA 15213

U.S.A.

E-mail: jkrueger@andrew.cmu.edu

URL: http://www.andrew.cmu.edu/ jkrueger 